\title{
INVERTIBLE KNOT CONCORDANCES AND PRIME KNOTS
}

\author{
Se-Goo KIM
}

\begin{abstract}
Silver and Whitten proved that every knot in $S^{3}$ is invertibly concordant to a hyperbolic knot by a series of Nakanishi's construction. We prove that every knot in $S^{3}$ is invertibly concordant to a nonhyperbolic prime knot by a simple one step satellite construction.
\end{abstract}

\section{Introduction}

Kirby and Lickorish [1] showed that every knot in $S^{3}$ is concordant to a prime knot, equivalently, every concordance class contains a prime knot. Generalizations appear in [3, 4, 5, 11]. Sumners [13] introduced the notion of invertible concordance. Nakanishi [6] strengthened Kirby and Lickorish's result by showing that every knot in $S^{3}$ is invertibly concordant to a prime knot with the same Alexader polynomial. Silver and Whitten [10] proved that a hyperbolic knot can be constructed by Nakanishi's method.

In contrast to this, we provide a one step method of satellite construction to show the following:

Theorem 1.1. Every knot in $S^{3}$ is invertibly concordant to a nonhyperbolic prime knot with the same Alexander polynomial by a one step satellite construction.

Corresponding to invertible concordance there is a group, the double concordance group, studied in $[2,7,12]$. A consequence of our work is that every double concordance class contains a nonhyperbolic prime knot.

Received March 5, 2010. Accepted March 15, 2010.

2000 Mathematics Subject Classification: 57M25.

Key words and phrases: Invertible knot concordance; Prime knots; Satellite construction. 


\section{Definitions and basic results}

In what follows manifolds and maps will be smooth and orientable. Let $I$ denote the interval $[0,1]$.

A link of $n$ components, $L$, is a smooth pair $\left(S^{3}, l\right)$ where $l$ is a smooth oriented submanifold of $S^{3}$ diffeomorphic to $n$ disjoint copies of $S^{1}$. A knot $K$ is a link of one component. Two links, $L_{1}$ and $L_{2}$, each of $n$ components, are called concordant if there exists a proper smooth oriented submanifold $w$ of $S^{3} \times I$, with $\partial w=\left(l_{1} \times 0\right) \cup\left(-l_{2} \times 1\right)$ and $w$ diffeomorphic to $n$ disjoint copies of $S^{1} \times I$. Let $\left(W ; L_{1}, L_{2}\right)$ denote $\left(S^{3} \times I, w\right)$ the concordance between $L_{1}$ and $L_{2}$. If $\left(W_{1} ; L_{1}, L_{2}\right)$ and $\left(W_{2} ; L_{2}, L_{3}\right)$ are two concordances with a common boundary component (oriented oppositely) we can then paste $W_{2}$ to $W_{1}$ along $L_{2}$ to get $\left(W_{1} \cup\right.$ $\left.W_{2} ; L_{1}, L_{3}\right)$.

A concordance $\left(W ; L_{1}, L_{2}\right)$ is said to be invertible at $L_{2}$ if there is a concordance $\left(W^{\prime} ; L_{2}, L_{1}\right)$ such that $\left(W \cup W^{\prime} ; L_{1}, L_{1}\right)$ is diffeomorphic to $\left(L_{1} \times I ; L_{1}, L_{1}\right)$, the product concordance of $L_{1}$. Given the above situation, we say that $L_{1}$ is invertibly concordant to $L_{2}$, and $L_{2}$ splits $L_{1} \times I$. In the same manner, concordance and invertible concordance can be defined for knots and links in the solid torus $S^{1} \times D^{2}$.

A submanifold $N$ with boundary is said to be proper in a manifold $M$ if $\partial N=N \cap \partial M$. Let $B^{3}$ denote the standard closed 3-ball $\left\{x \in \mathbb{R}^{3} \mid\right.$ $|x| \leq 1\}$. An $n$-tangle $T$ is a smooth pair $\left(B^{3}, \lambda\right)$ where $\lambda$ is a proper embedding of $n$ disjoint copies of the interval $I$ into $B^{3}$. Throughout this paper, an embedding means either the map or the image. Let $U_{n}$ denote a trivial $n$-tangle, i.e., $U_{n}$ consists of $n$ unlinked unknotted arcs. For example, $U_{1}$ is the unknotted standard ball pair $\left(B^{3}, I\right)$. For $n=2$, see Figure 1.

Concordances and invertible concordances between tangles can be defined in a similar way as for links. However, the boundary of the 3-ball $B^{3}$ is required to be fixed at each stage of concordance. More precisely, let $I_{1}, \ldots, I_{n}$, denote $n$ disjoint copies of the interval $I$. Two $n$-tangles, $T_{0}=\left(B^{3}, \lambda_{0}\right)$ and $T_{1}=\left(B^{3}, \lambda_{1}\right)$, are concordant if there is a proper smooth embedding $\tau$ of $\left(\cup_{i=1}^{n} I_{i}\right) \times I$ into $B^{3} \times I$, with $\tau\left(\cup_{i=1}^{n} I_{i} \times \epsilon\right)=$ $\lambda_{\epsilon}(\epsilon=0,1)$ and $\tau\left(\epsilon_{i} \times I\right)=\tau\left(\epsilon_{i} \times 0\right) \times I$ for each $i=1, \ldots, n$, and $\epsilon_{i}=0,1$ in $I_{i}$. Let $\left(V ; T_{1}, T_{2}\right)$ denote $\left(B^{3} \times I, \tau\right)$, the concordance between $T_{1}$ and $T_{2}$. If $\left(V ; T_{1}, T_{2}\right)$ and $\left(V^{\prime} ; T_{2}, T_{3}\right)$ are two concordances, we can then paste $V^{\prime}$ to $V$ along $T_{2}$ to get a concordance $\left(V \cup V^{\prime} ; T_{1}, T_{3}\right)$. A concordance $\left(V ; T_{1}, T_{2}\right)$ is invertible at $T_{2}$ if there is a concordance $\left(V^{\prime} ; T_{2}, T_{1}\right)$ such that $\left(V \cup V^{\prime} ; T_{1}, T_{1}\right)$ is diffeomorphic to $\left(T_{1} \times I ; T_{1}, T_{1}\right)$ 
by a diffeomorphism $\varphi$ with $\varphi(\tau)=\lambda_{1} \times I$, where $\tau$ is the embedding of $n$ disjoint copies of $I \times I$ into $B^{3} \times I$ defining the concordance $\left(V \cup V^{\prime} ; T_{1}, T_{1}\right)$ and $\lambda_{1}$ is the embedding of $n$ disjoint copies of $I$ into $B^{3}$ defining the tangle $T_{1}$.

A knot is called doubly null concordant if it is the slice of some unknotted 2-sphere in $S^{4}$. Two knots $K_{1}$ and $K_{2}$ are said to be doubly concordant if $K_{1} \# J_{1}$ is isotopic to $K_{2} \# J_{2}$ for some doubly null concordant knots $J_{1}$ and $J_{2}$.

The following theorem is due to Zeeman.

Theorem 2.1. [14] Every 1-twist-spun knot is unknotted.

Let $-K$ denote the knot obtained by taking the image of $K$, with reversed orientation, under a reflection of $S^{3}$. The following fact was first proved by Stallings and now follows readily from 2.1. (One cross-section of the 1-twist-spin of $K$ yields $K \#(-K)$. For details, see [13].) $K$.

Corollary 2.2. $K \#(-K)$ is doubly null concordant for every knot

Corollary 2.3. If $K_{1} \#\left(-K_{2}\right)$ is doubly null concordant then $K_{1}$ and $K_{2}$ are doubly concordant.

Proof. Take $J_{1}=K_{2} \#\left(-K_{2}\right)$ and $J_{2}=K_{1} \#\left(-K_{2}\right)$ in the definition of double concordance.

Remark 2.4. An easy exercise shows that knots $K_{1}$ and $K_{2}$ are concordant if and only if $K_{1} \#\left(-K_{2}\right)$ is slice, i.e., concordant to the unknot. This defines an equivalence relation. However, a definition of double concordance more along the lines of concordance is as of yet inaccessible. The difficulty is that it is unknown whether the following is true: If knots $K$ and $K \# J$ are doubly null concordant, then $J$ is doubly null concordant.

There is a relation between invertible concordance and double concordance.

Proposition 2.5. If $K_{1}$ is invertibly concordant to $K_{2}$ then $K_{1} \#\left(-K_{2}\right)$ is doubly null concordant.

Proof. There is a copy of $S^{3} \times I$ in $S^{4}$ intersecting the 1-twist-spin of $K_{1}$ in $K_{1} \#\left(-K_{1}\right) \times I$. Since $K_{2}$ splits $K_{1} \times I$, there is an invertible concordance from $K_{1} \#\left(-K_{1}\right)$ to $K_{1} \#\left(-K_{2}\right)$. Hence $K_{1} \#\left(-K_{1}\right) \times I$ is split by $K_{1} \#\left(-K_{2}\right)$ and the result follows. 


\section{Invertible concordances and prime knots}

Kirby and Lickorish [1] proved that any knot in $S^{3}$ is concordant to a prime knot. Livingston [3] gave a different proof of this result using satellite knots. In this section, we modify Livingston's approach to prove Theorem 1.1.

Before proving this, we will set up some notation. By a splitting- $S^{2}$, $S$, for a knot $K$ (in $S^{3}$ or $S^{1} \times D^{2}$ ) we denote an embedded 2-sphere, $S$, intersecting $K$ in exactly 2 points. A knot in either $S^{3}$ or $S^{1} \times D^{2}$ is prime if for every splitting- $S^{2}, S, S$ bounds some 3 -ball, $B$, with $(B, B \cap K)$ a trivial pair. The winding number of a knot $K$ in $S^{1} \times D^{2}$ is that element $z$ of $\mathbb{Z} \cong H_{1}\left(S^{1} \times D^{2} ; \mathbb{Z}\right)$ with $z \geq 0$ and $K$ representing $z$. The wrapping number of $K$ is the minimum number of intersections of $K$ with a disk $D$ in $S^{1} \times D^{2}$ with $\partial D=$ meridian. If $K_{1}$ is a knot in $S^{1} \times D^{2}$ and $K_{2}$ is a knot in $S^{3}$, the $K_{1}$ satellite of $K_{2}$ is the knot in $S^{3}$ formed by mapping $S^{1} \times D^{2}$ into the regular neighborhood of $K_{2}$, $N\left(K_{2}\right)$, and considering the image of $K_{1}$ under this map. The only restriction on the map of $S^{1} \times D^{2}$ into $N\left(K_{2}\right)$ is that it maps a meridian to a meridian. In what follows we will consider $S^{1} \times D^{2}$ embedded in $S^{3}$ in a standard way. Hence any $\operatorname{knot} K$ in $S^{1} \times D^{2}$ gives rise to a knot $K^{*}$ in $S^{3}$.

The following theorem is due to Livingston.

Theorem 3.1. [3] Let $K_{1}$ be a knot in $S^{1} \times D^{2}$ such that $K_{1}^{*}$ is the unknot in $S^{3}$. Then $K_{1}$ is prime in $S^{1} \times D^{2}$. Moreover, if $K_{1}$ has wrapping number $>1$ and $K_{2}$ is any nontrivial knot in $S^{3}$, then the $K_{1}$ satellite of $K_{2}$ is prime in $S^{3}$.

This theorem suggests that, to prove our main theorem 1.1, we only need to find a knot $K_{1}$ in $S^{1} \times D^{2}$ with $K_{1}^{*}$ the unknot in $S^{3}$ and an invertible concordance between the core $C$ and the knot $K_{1}$ in $S^{1} \times D^{2}$. To do this, we observe that there is an invertible concordance between the tangles $U_{2}$ and $T$ in Figure 1. We remark here that Ruberman in [8] has used the tangle $T$ to prove that any closed orientable 3 -manifold is invertibly homology cobordant to a hyperbolic 3-manifold.

Lemma 3.2. The 2-tangle $T$ in Figure 1(b) splits $U_{2} \times I$.

Proof. Let $I_{1}$ be a copy of the non-straight arc of $T$ in the 3-ball $B^{3}$ and let $J_{1}$ be a copy of the non-straight arc of $U_{2}$ in $B^{3}$ as shown in Figure 1(c). The closed curve $J_{1} \cup I_{1}$ bounds an obvious punctured torus $F$ that is the shaded region in Figure 1(c). Consider $F$ as the plumbing 


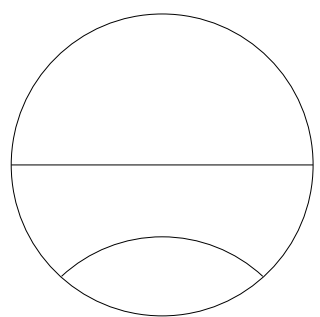

(a) $U_{2}$

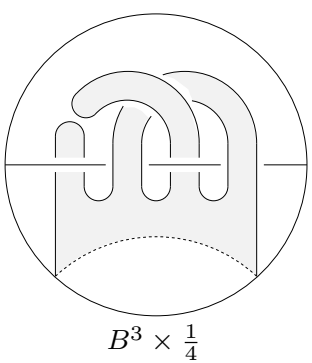

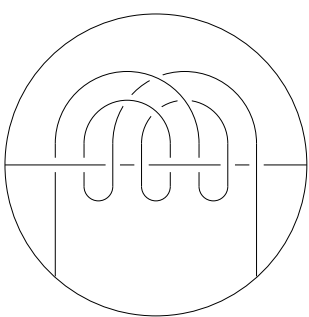

(b) $T$

FIGURE 1
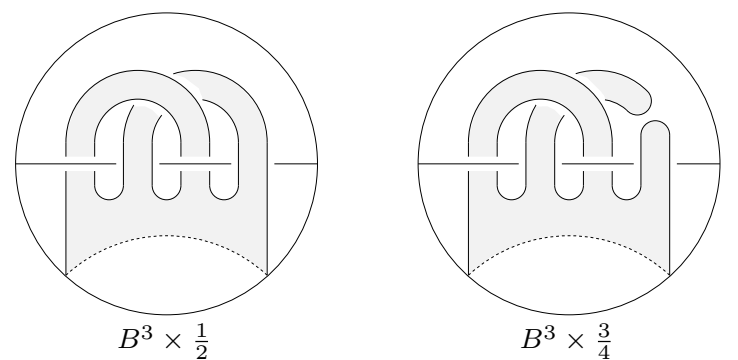

FiguRe 2

of two $S^{1} \times I$. Let $c_{i}, i=1,2$, be the cores of the two $S^{1} \times I$ of $F$ and let $\bar{c}_{i}, i=1,2$, be disjoint proper line segments in $F$ intersecting with $c_{i}$ exactly once, respectively. See Figure 1(c).

To construct an invertible concordance, we will construct two concordances and then paste them together. First, note that pinching $I_{1}$ along $\bar{c}_{1}$ transforms $T$ into the tangle $U_{2}$ with an unlinked unknotted circle inside which is isotopic to the circle $c_{2}$. Now capping off this circle we have a concordance $\left(V_{1}^{\prime} ; T, U_{2}\right)$. The tangle $B^{3} \times \frac{1}{4}$ in Figure 2 represents a slice of this concordance before capping off the circle. In the similar way, pinching $I_{1}$ along $\bar{c}_{2}$ and capping off the unknot gives us another concordance $\left(V_{2} ; T, U_{2}\right)$. Let $\left(V_{1} ; U_{2}, T\right)$ denote the concordance $\left(V_{1}^{\prime} ; T, U_{2}\right)$ with reversed orientation. We can then paste $V_{1}$ to $V_{2}$ along $T$ to get a concordance $\left(V_{1} \cup V_{2} ; U_{2}, U_{2}\right)$, which will be proved to be isotopic to the product concordance $U_{2} \times I$. A few cross-sections of concordance $V_{1} \cup V_{2}$ are drawn in Figure 2 .

Let $\tau$ denote the embedding of two disjoint copies of $I \times I$ into $V_{1} \cup V_{2}$ as in the definition of concordance in Section 2. It is obvious from Figure 2 that there is a 3 -manifold $M$ (the union of shaded regions) in $V_{1} \cup V_{2}$ bounded by $\tau$ and $J_{1} \times I$, whose intersection with $U_{2}$ at each end 


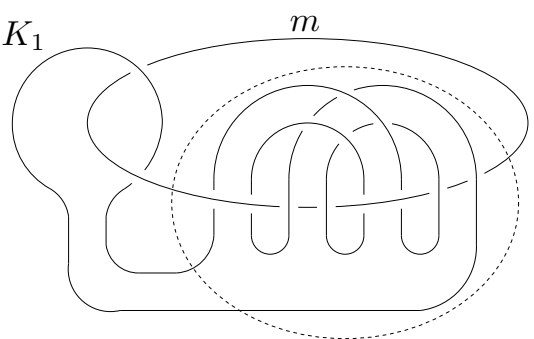

(a)

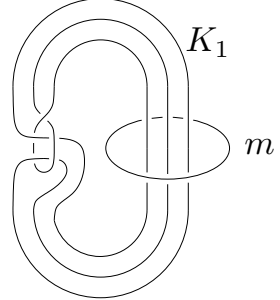

(c)

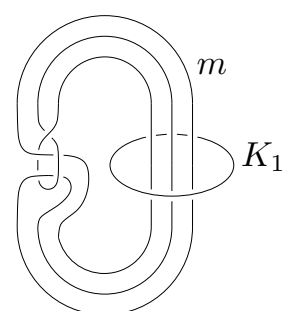

(d)

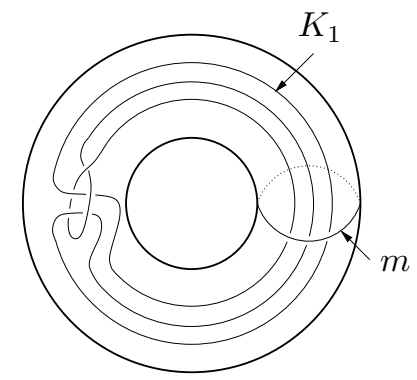

(b)

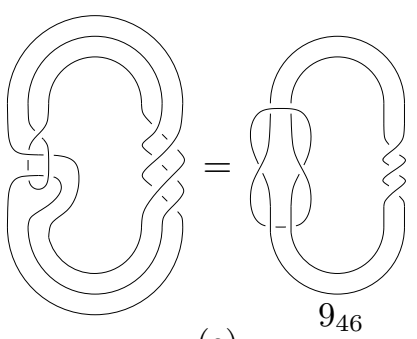

(e)

Figure 3

of the concordance is the arc $J_{1}$ and whose cross-section in the middle is the punctured torus $F$. This 3-manifold $M$ can be considered as the union of three submanifolds: the product $F \times I$ and two 3-dimensional 2handles $D^{2} \times I$. One $D^{2} \times I$ is glued to $F \times I$ along a regular neighborhood of $c_{2}$, which corresponds to capping off the circle isotopic to $c_{2}$ as we constructed the concordance $V_{1}^{\prime}$. The other $D^{2} \times I$ is glued along a regular neighborhood of $c_{1}$, which corresponds to capping off the circle isotopic to $c_{1}$ as we constructed the concordance $V_{2}$. Since $F \times I$ is a 3 -dimensional handlebody with 2 handles with cores $c_{1}$ and $c_{2}, M$ is the manifold that results by adding two 2-handles to a genus 2 solid handlebody along the cores of the 1-handles, in this case yielding $B^{3}$. Moreover, $M$ does not intersect the other straight arc of $T$ at any stage. Using this 3 -ball $M$, we can isotop $\tau$ to $J_{1} \times I$ in a regular neighborhood of $M$ not disturbing the other arc and $\partial B^{3}$. This completes the proof.

Proposition 3.3. The knot $K_{1}$ in Figure $3(b)$ splits $C \times I$, where $C$ is the core in $S^{1} \times D^{2}$.

Proof. Consider $S^{1} \times D^{2}$ as the complement of the unknot $m$ in $S^{3}$. The knot $K_{1}$ in Figure 3(b) is isotopic to $K_{1}$ in Figure 3(a). It is obvious from Figure 3(a) that $K_{1} \cup m$ is the link in $S^{3}$ formed by replacing a 
trivial 2-tangle in Hopf link with $T$ (dotted circle in Figure 3(a)). The proposition now follows from Lemma 3.2.

Now we are ready to prove our main theorem 1.1.

Proof of Theorem 1.1. Let $K$ be a knot in $S^{3}$. If $K$ is trivial, it is prime itself. Suppose now that $K$ is nontrivial. Let $K^{\prime}$ be $K_{1}$ satellite of $K$ where $K_{1}$ is the knot in $S^{1} \times D^{2}$ in Figure 3(b). By Proposition 3.3, $K^{\prime}$ splits $K \times I$. We now only need to show that $K^{\prime}$ is prime. Since $K_{1}^{*}$ is the unknot in $S^{3}, K_{1}$ is prime by Theorem 3.1 and to complete this proof it remains to show its wrapping number $>1$. Its winding number is 1 , hence its wrapping number is at least one. It is easy to see that the core knot is the only prime knot in $S^{1} \times D^{2}$ with wrapping number 1 . So, if $K_{1}$ had wrapping number 1 , then it is isotopic to the core of $S^{1} \times D^{2}$. The -1 surgery on the meridian curve $m$ in $S^{3}$ should make $K_{1}^{*}$ unchanged, i.e., unknotted. However, the knot in Figure 3(e), the result of $K_{1}^{*}$ after -1 surgery along $m$, is $9_{46}$ and hence knotted. Therefore the wrapping number is greater than 1 .

Corollary 3.4. Any knot is doubly concordant to a prime knot.

Remark 3.5. The $K_{1}$ satellite of $K$ has the same Alexander polynomial as that of $K$. Seifert [9] proved that the Alexander polynomial of the $K_{1}$ satellite of $K$ is $\Delta_{K_{1}^{*}}(t) \Delta_{K}\left(t^{w}\right)$ if $w$ is the winding number of $K_{1}$ in $S^{1} \times D^{2}$. In our case, $w$ is 1 and $K_{1}^{*}$ is the unknot.

In [3], Livingston also proved that every 3-manifold is homology cobordant to an irreducible 3-manifold. Two 3-manifolds, $M_{1}$ and $M_{2}$, are homology cobordant if there is a 4-manifold $W$, with $\partial W=M_{1} \cup M_{2}$ and the map of $H_{*}\left(M_{i} ; \mathbb{Z}\right) \rightarrow H_{*}(W ; \mathbb{Z})$ an isomorphism. Invertible homology cobordisms can be defined in the same way as in the knot concordance case. A 3-manifold $M$ is irreducible if every embedded $S^{2}$ in $M$ bounds an embedded $B^{3}$.

Remark 3.6. In spirit of [3], we have a simple proof that every 3manifold is invertibly homology cobordant to an irreducible 3-manifold. To prove this, we only need to modify slightly the proof of Theorem 3.2 in [3] by using $K_{1}$ in Figure $3(\mathrm{~b})$. The -1 surgery on $K_{1}$ makes the meridian $m$ the knot $9_{46}$.

This remark is also a corollary of Ruberman's Theorem 2.6 in [8] that reads: for every closed orientable 3 -manifold $N$, there is a hyperbolic 3 manifold $M$, and an invertible homology cobordism from $M$ to $N$. The remark follows since a hyperbolic 3-manifold is irreducible. 


\section{Acknowledgements}

The author would like to thank Katura Miyazaki to inform of Nakanish's result. The author also thanks anonymous referees for careful reading of this paper and helpful comments. This work was supported by the National Research Foundation of Korea(NRF) grant funded by the Korea government(MEST) (No. 2009-0074487).

\section{References}

[1] R. C. Kirby and W. B. R. Lickorish, Prime knots and concordance, Math. Proc. Cambridge Philos. Soc. 86 (1979), 437-441.

[2] J. Levine, Doubly sliced knots and doubled disk knots, Michigan Math. J. 30 (1983), no. 2, 249-256.

[3] C. Livingston, Homology cobordisms of 3-manifolds, knot concordances, and prime knots, Pacific J. Math. 94 (1981), no. 1, 193-206.

[4] R. Myers, Homology cobordisms, link concordances, and hyperbolic 3-manifolds, Trans. Amer. Math. Soc. 278 (1983), no. 1, 271-288.

[5] R. Myers, Excellent 1-manifolds in compact 3-manifolds, Topology Appl. 49 (1993), no. 2, 115-127.

[6] Y. Nakanishi, Primeness of links, Math. Sem. Notes Kobe Univ. 9 (1981), 415440.

[7] D. Ruberman, Doubly slice knots and the Casson-Gordon invariants, Trans. Amer. Math. Soc. 279 (1983), no. 2, 569-588.

[8] D. Ruberman, Seifert surfaces of knots in $S^{4}$, Pacific J. Math. 145 (1990), no. 1, 97-116.

[9] H. Seifert, On the homology invariants of knots, Quart. J. Math. Oxford Ser. (2) 1 (1950), 23-32.

[10] D. Silver and W. Whitten, Hyperbolic covering knots, Algebr. Geom. Topol. 5 (2005), 1451-1469 (electronic).

[11] T. Soma, Hyperbolic, fibred links and fibre-concordances, Math. Proc. Cambridge Philos. Soc. 96 (1984), no. 2, 283-294.

[12] N. W. Stoltzfus, Algebraic computations of the integral concordance and double null concordance group of knots, Knot theory (Proc. Sem., Plans-sur-Bex, 1977), pp.274-290, Lecture Notes in Math. 685, (J.C. Hausmann, ed.), Springer-Verlag, Berlin, 1978.

[13] D. W. Sumners, Invertible knot cobordisms, Comment. Math. Helv. 46 (1971), 240-256.

[14] E. C. Zeeman, Twisting spun knots, Trans. Amer. Math. Soc. 115 (1965), 471495. 
Department of Mathematics, School of Science, Kyung Hee University,

1 Hoegi-dong, Dongdaemun-gu, Seoul 130-701, Republic of Korea

E-mail: sgkim@khu.ac.kr 